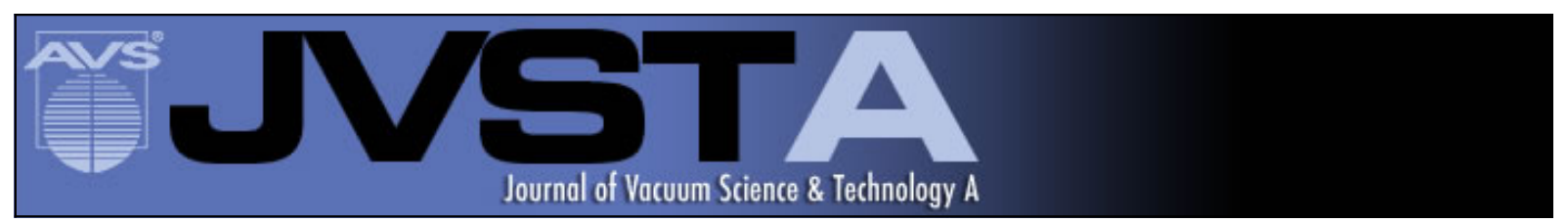

\title{
Atomically precise surface engineering of silicon CCDs for enhanced UV quantum efficiency
}

Frank Greer, Erika Hamden, Blake C. Jacquot, Michael E. Hoenk, Todd J. Jones, Matthew R. Dickie, Steve P. Monacos, and Shouleh Nikzad

Citation: Journal of Vacuum Science \& Technology A 31, 01 A103 (2013); doi: 10.1116/1.4750372

View online: http://dx.doi.org/10.1116/1.4750372

View Table of Contents: http://scitation.aip.org/content/avs/journal/jvsta/31/1?ver=pdfcov

Published by the AVS: Science \& Technology of Materials, Interfaces, and Processing

\section{Articles you may be interested in}

Atomic layer deposited high- nanolaminates for silicon surface passivation

J. Vac. Sci. Technol. B 32, 03D110 (2014); 10.1116/1.4863499

Extremely low surface recombination velocities in black silicon passivated by atomic layer deposition Appl. Phys. Lett. 100, 191603 (2012); 10.1063/1.4714546

Enhancement in the efficiency of light emission from silicon by a thin Al 2 O 3 surface-passivating layer grown by atomic layer deposition at low temperature

J. Appl. Phys. 101, 033130 (2007); 10.1063/1.2464190

Enhanced quantum efficiency of high-purity silicon imaging detectors by ultralow temperature surface modification using $\mathrm{Sb}$ doping

Appl. Phys. Lett. 87, 254101 (2005); 10.1063/1.2149181

Substrate preparation and low-temperature boron doped silicon growth on wafer-scale charge-coupled devices by molecular beam epitaxy

J. Vac. Sci. Technol. B 20, 1170 (2002); 10.1116/1.1477200

\section{HIDEN}

\section{Instruments for Advanced Science}

w www.HidenAnalytical.com w www.HidenAnalytical.com E info@hiden.co.uk CLICK TO VIEW our product catalogue

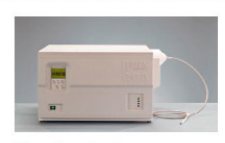

Gas Analysis , dynamic measurement of reaction gas streams catalysis and thermal analysis , molecular beam studies
, dissolved species probes , dissolved species probes
, fermentation, environmental and ecological studies

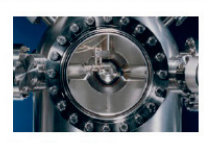
Surface Science , UHVTPD

, SIMS
, end point detection in ion beam etch , end point detection in ion beam etch
, elemental imaging - surface mapping

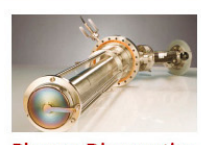

Plasma Diagnostics , plasma source characterization etch and deposition process reaction analysis of neutral and radical species

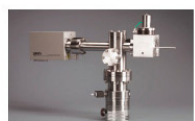

Vacuum Analysis , partial pressure measurement and contro of process gases reactive sputter process control vacuum diagnostics 


\title{
Atomically precise surface engineering of silicon CCDs for enhanced UV quantum efficiency
}

\author{
Frank Greer ${ }^{a)}$ \\ Jet Propulsion Laboratory, California Institute of Technology, 4800 Oak Grove Drive, Pasadena, \\ California 91109 \\ Erika Hamden \\ Jet Propulsion Laboratory, California Institute of Technology, 4800 Oak Grove Drive, Pasadena, California \\ 91109 and Department of Astronomy, Columbia University, 550 W 120th St, New York, New York 10025 \\ Blake C. Jacquot, ${ }^{\text {b) }}$ Michael E. Hoenk, Todd J. Jones, Matthew R. Dickie, Steve P. Monacos, \\ and Shouleh Nikzad \\ Jet Propulsion Laboratory, California Institute of Technology, 4800 Oak Grove Drive, Pasadena, \\ California 91109
}

(Received 1 August 2012; accepted 21 August 2012; published 13 September 2012)

\begin{abstract}
The authors report here on a new technique, combining the atomic precision of molecular beam epitaxy and atomic layer deposition, to fabricate back illuminated silicon CCD detectors that demonstrate world record detector quantum efficiency $(>50 \%)$ in the near and far ultraviolet $(155-300 \mathrm{~nm})$. This report describes in detail the unique surface engineering approaches used and demonstrates the robustness of detector performance that is obtained by achieving atomic level precision at key steps in the fabrication process. The characterization, materials, and devices produced in this effort will be presented along with comparison to other approaches. (C) 2013 American Vacuum Society. [http://dx.doi.org/10.1116/1.4750372]
\end{abstract}

\section{INTRODUCTION}

The ultraviolet (UV) represents an extremely important region of the electromagnetic spectrum as it has a critical role in a wide variety of scientific, commercial, and government applications. For example, ultraviolet spectroscopy can be utilized to study planetary atmospheres to determine whether basic elements for life such as oxygen, nitrogen, and hydrogen are present. ${ }^{1}$ A UV spectrometer can also detect evidence of small quantities of complex organic molecules (e.g., tholins) from the UV reflectivity of the surface of an icy moon, providing information on how prebiotic chemistry takes place on extraterrestrial bodies. ${ }^{2}$ High sensitivity astronomical observations in the UV regime could enable the observation of faint emission from the intergalactic medium, which likely represent $50 \%$ of the detectable baryonic mass in the universe. ${ }^{3}$ Recent measurements enabled by the first all sky UV survey mission, GALEX, have uncovered a startling comet-like tail behind a red giant star that is streaking through space at nearly 300000 miles/h. This phenomenon is unique and can only be observed in the UV and has now provided a means to characterize how stars die and ultimately seed new solar systems through the shedding of carbon, oxygen, and other elements. ${ }^{4}$ Extreme UV lithography is utilized to pattern the finest features of the latest generation of semiconductor devices. UV laser inspection and imaging is therefore critical to identify defects in the fabrication process to maximize yield and reduce cost in this highly competitive industry. ${ }^{5} \mathrm{UV}$ imaging has also recently been used in medi-

\footnotetext{
${ }^{a)}$ Electronic mail: frank.greer@jpl.nasa.gov

${ }^{b}$ Present address: The Aerospace Corporation, 2310 E. El Segundo Blvd., El Segundo, CA 90245-4609.
}

cal imaging to study how caffeine affects calcium ionic pathways in the brain. ${ }^{6}$ Rockets produce significant UV emission due to the production of excited nitrogen oxide species in their plumes. ${ }^{7}$ While infrared imaging is clearly an important antimissile defense technology, UV can offer significant advantages in this application due to the ability to observe even in direct sunlight using "solar-blind" imaging. Bite marks can be readily observed and identified in forensic investigations since human saliva (wet or dry) shines brightly under UV illumination. ${ }^{8}$ Bruises are also evident for many days in UV after they have disappeared to the naked eye. As this only represents a small fraction of the real world applications of UV detectors, there is clearly strong motivation to have detectors with the highest possible sensitivity.

Unfortunately, despite this wide range of applications, scientific imaging in the ultraviolet is extremely difficult because the technology for sensing UV light is substantially limited by the quantum efficiency $(\mathrm{QE})$ of available detectors and the transparency of optical coating materials. Many materials absorb near and far UV light so strongly that thicknesses of 20 nanometers or less are completely opaque. Thus, the UV throughput of an instrument is highly sensitive to impurities on, or contained in, any of the optical elements or the detector itself. Compounding this problem is that many important sources of UV light are faint, so maximizing detector sensitivity is critical to unlocking the true potential of UV imaging. Table I outlines three examples of ultraviolet sensitive detectors that are currently in use or have been used in space missions along with their typical quantum efficiencies in the near and far UV. ${ }^{9,10}$ New classes of III-nitride materials based on molecular beam epitaxy (MBE) or metal organic chemical vapor deposition (MOCVD) grown $\mathrm{GaN}$ or $\mathrm{AlGaN}$ hold significant promise for future generations of 
TABLE I. Performance of current typical UV detectors in major space missions.

\begin{tabular}{lcc}
\hline \hline & Typical quantum efficiency(155-300 $\mathbf{n m})$ & Example of use in astronomy \\
\hline $\mathbf{C s}_{\mathbf{2}}$ Te microchannel plates & $\sim 10 \%$ or less & GALEX Space Telescope \\
Silicon CCD coated with Lumogen & $\sim 15-25 \%$ or less & Cassini ISS, WF/PC2-Hubble Space Telescope \\
Ion implanted-laser annealed CCD & $\sim 15-25 \%$ or less & WF3-Hubble Space Telescope \\
\hline \hline
\end{tabular}

UV detectors, but they still face materials challenges to achieve what is intrinsically possible theoretically. Silicon based charge coupled devices (CCDs), however, are based on the same fabrication technologies utilized in the semiconductor industry and are heavily used in commercial imaging applications such as digital cameras. Silicon-CMOS (complementary metal oxide semiconductor) based imagers are also widespread, and their use in commercial and scientific applications has been accelerating over the last few years due to the rapid progress of the overall CMOS industry. Unfortunately, the native oxide that naturally forms on silicon causes unfavorable bending in silicon's electronic band structure near the surface. This leads to the capture of UV-produced photoelectrons in surface traps and leads to instabilities and very poor response below $400 \mathrm{~nm}$ for silicon imagers. ${ }^{11}$ This can be overcome though a combination of techniques known as back illumination and back surface passivation.

Commercial methods, such as chemisorption and ion implant/laser anneal, do exist to passivate the back surface of silicon CCDs. ${ }^{12-14}$ These techniques work well in the visible and near UV; however, they have limitations in the shorter wavelengths in terms of spectral range, sensitivity, response stability, and hysteresis. Delta-doping using silicon $\mathrm{MBE}$, on the other hand, enables precise control over the band structure at the CCD surface to get ideal silicon reflection-limited response (see Fig. 1). ${ }^{15,16}$

Even with near ideal back surface passivation, the inherent reflectivity of silicon significantly limits the absolute detector quantum efficiency of silicon CCDs. ${ }^{16}$ This is illustrated by the dip in quantum efficiency to $\sim 25 \%$ near $280 \mathrm{~nm}$ in Fig. 1 . Therefore, antireflection (AR) coatings should be utilized to maximize imaging performance. Modeling results predict that absolute quantum efficiencies of $>50 \%$ should be achievable in the near and far UV $(100-300 \mathrm{~nm}) .{ }^{17}$ We define absolute quantum efficiency here as the probability of a photon incident at the detector surface will generate one or more electrons that are detected by the detector element. This is to be distinguished from internal quantum efficiency which subtracts out reflection losses and therefore only considers those photons which have the opportunity to be absorbed by the detector. Note that single photons at very short wavelengths can produce more than one electron when they are absorbed by a silicon detector. ${ }^{18}$ Therefore, we correct our calculations of absolute quantum efficiency for this gain factor, taking into account that the quantum yield for those photons is greater than unity.

It should be noted that AR coatings are widely utilized for many detector systems (silicon, III-V, etc.) in the visible and infrared to improve absolute quantum efficiency.
However, producing UV antireflection coatings is challenging as the coatings must be high quality (i.e., low in impurity/defect concentration to avoid UV absorption and pinhole free to prevent humidity interaction with the imager surface). In addition, the index of refraction of silicon varies over the UV, and therefore, multiple materials are required to cover the near and far UV effectively. This is especially important because even ideal materials, such as hafnium oxide, have absorption cutoffs that make them opaque in the far ultraviolet (FUV) (in this case, below $240 \mathrm{~nm}$ ). In addition, a thickness change of $2 \mathrm{~nm}$ or less can shift the peak antireflection performance of a coating or lead to dramatic changes in absorption cutoffs, especially in the far UV, making controllable and reproducible fabrication of ultrahigh performance AR coated silicon CCDs difficult. Recent modeling results by Hamden et al. have demonstrated that the target thicknesses for UV antireflective coatings range from 10 to $25 \mathrm{~nm} .{ }^{17}$ When all of these constraints are considered together, it is clear that a series of robust, nanoscale, surface-engineering processes are required to produce the best possible UV-sensitive CCDs. In this study, we have used back-illumination, silicon bandstructure engineering/surface passivation, and precision AR coating techniques, including atomic layer deposition (ALD), to achieve this end.

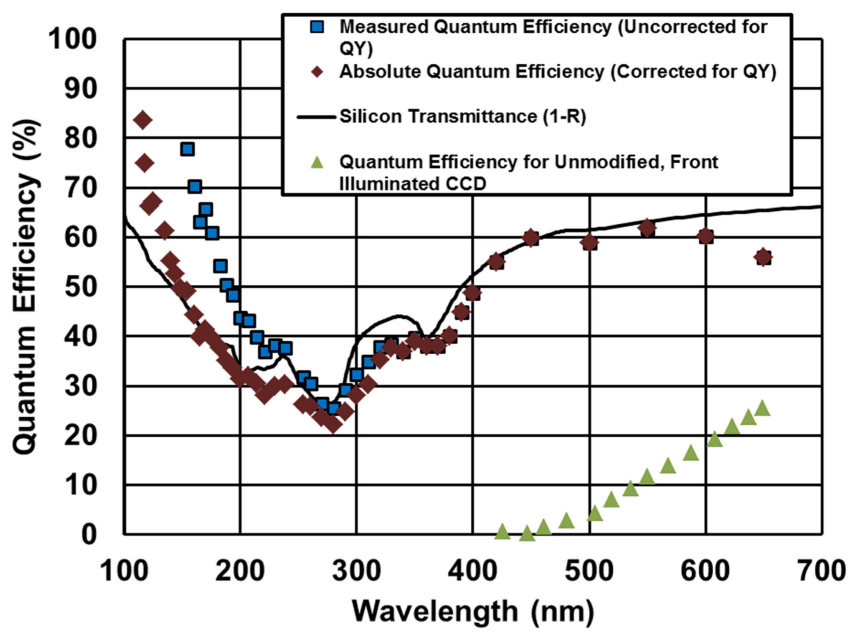

FIG. 1. (Color online) Measured and absolute quantum efficiency for a backilluminated, delta-doped silicon CCD. This CCD was not AR coated. Note that the data, once corrected for quantum yield, lies along the silicon transmittance curve. This indicates that the CCD is exhibiting reflection-limited response and $100 \%$ internal quantum efficiency. Data for an unmodified, front-illuminated $\mathrm{CCD}$ is shown for comparison purposes to illustrate the improvement in UV sensitivity that is achieved by the delta-doping and back-illumination processes. 


\section{EXPERIMENT}

Wafers containing fully fabricated CCDs of several types were acquired for this investigation from multiple commercial sources. These included: (1) flight spare CCD wafers, originally manufactured by Loral to supply the main camera on board the Cassini spacecraft mission. This mission, launched in 1997, is currently flying in the Saturn system, ${ }^{19}$ (2) CCD imagers obtained from EG\&G Reticon, and (3) wafers containing newly designed electron-multiplied CCDs (EM-CCDs) from e2v. These EM-CCDs have a gain register, which results in measurements with extremely high signal to noise ratio (as high as 10 000:1), enabling them to be utilized in photon counting applications. These three different types CCDs were utilized to demonstrate the robustness of the techniques in this investigation and show that this is a generally applicable approach to enhance the UV quantum efficiency of any type of silicon imager.

The CCDs were set up for back-illumination to remove the frontside circuitry from the photon pathway. The bulk thickness of the CCDs is reduced by polishing and chemical etching to leave a thin membrane. The final thickness of the membrane after this process is thick enough so that it has sufficient mechanical strength to withstand its environment. The membrane is also thin enough so that charge carriers produced at the back surface do not diffuse too far laterally as they drift to their destination pixel on the frontside. This maps the point of photon entry to a specific frontside pixel in the image. CCDs are produced with an epitaxial layer that provides a natural definition for the membrane thickness. It is uniform across the entire CCD and provides a chemical etch-stop for the thinning process. For this work, the membrane is created in a way that makes it monolithic with a thick frame of original silicon from the CCD. In fact, the frontside CCD circuitry resides, in continuity, over regions that are thick frame and thin membrane. The thick frame serves the purpose of mechanically supporting the membrane, and it makes it convenient to wire bond the imaging device using its existing bond pads. It should be noted that residual stresses in the imaging membrane result in macroscale deformations when the frame thinning approach is used (see Fig. 2). However, this frame thinning approach is a relatively easy and inexpensive way to back-illuminate a silicon CCD and therefore is ideal for proof of concept demonstrations. Backside illumination processes where the entire CCD is thinned are currently commercially available, but these commercial processes are not currently compatible with the MBE and ALD techniques that will be discussed in this report. For these experiments, the quick approach of frame thinning was used. An approach where the entire CCD is thinned that is compatible with MBE and ALD has been developed at JPL.

In the frame thinning approach, the backside of the $\mathrm{CCD}$ wafer is polished by chemical mechanical polishing (CMP), and the membrane region is defined by a nitride mask on the polished surface. The bulk silicon is aggressively etched by hot $55 \% \mathrm{KOH}$ except for the frame defined by the nitride mask. We monitor the progress of the etch and terminate

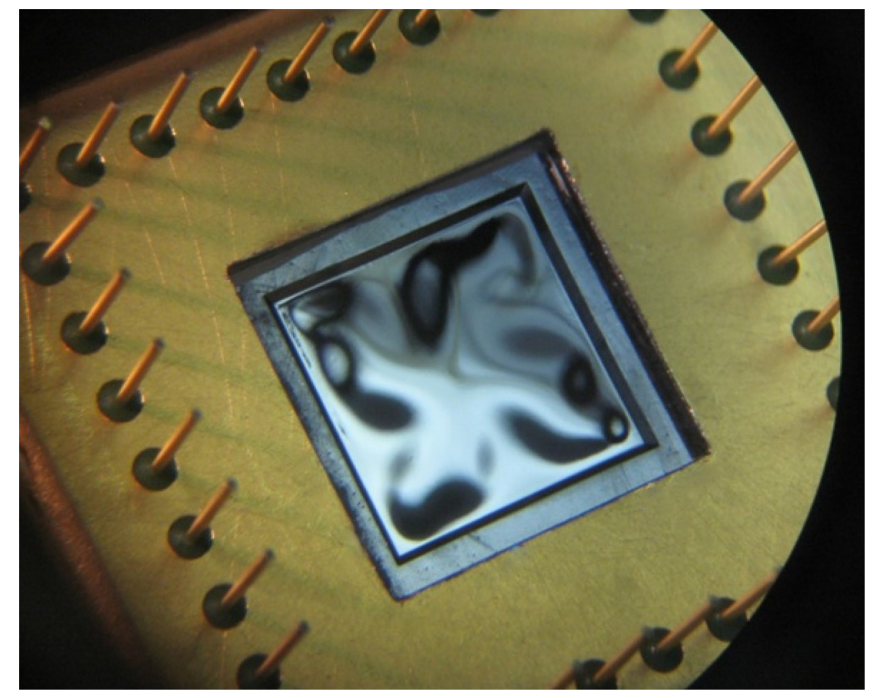

FIg. 2. (Color online) Cassini CCD that has been frame thinned to enable back-illumination for proof-of-concept demonstration of ultrahigh absolute UV quantum efficiency. Its frontside detection electronics are face down as pictured. This CCD is mounted in a picture frame package with a window cut to enable the backside illumination. The clover leaf pattern evident in the thin membrane is due to compressive stresses present in the processed silicon. It should be noted that an alternative thinning approach, currently under development at JPL, involves the use of a handle wafer to support the imaging membrane. This approach eliminates the deformation and is appropriate for high volume production of scientific grade CCDs.

when it comes within $20 \mu \mathrm{m}$ of the known epitaxial layer thickness. The surface texture of the $\mathrm{KOH}$ etch is rough by comparison to the CMP surface. This condition is rectified by the next step by isotropic etching in a specific mixture of hydrofluoric, nitric, and acetic acids (HNA). The expression for relative volume in the mixture is 1:3:8. This mixture of HNA continues the etch to the epitaxial layer where it selfterminates upon exhaustion of bulk silicon. The chemical reason for the well defined etch stop comes from the higher resistivity of the epitaxial layer. The epitaxial layer has many orders of magnitude fewer charge carriers on hand for participation in the chemical reaction of the etch. The etch reaction stops as it is starved for charge carriers. ${ }^{20}$ Additional brief etch exposures are made to mixtures of 1:40:15 $\mathrm{HNA}$ and $\mathrm{KMnO}_{4} / \mathrm{HF}$. These solutions help to remove certain stains and haze, which are left by the previous reactions. The resulting thin membrane has a smooth mirror-like specular finish.

To prepare the CCDs for electrical passivation and band structure engineering, they are brought into an inert and clean glove box environment. A UV ozone clean is utilized to remove adventitious carbon and other organics, and a HFethanol spin clean is utilized to remove the native oxide from the imaging membrane. The backside surface of the imager is then passivated by delta-doping. ${ }^{11,21,22}$ Briefly, in delta-doping, an extremely thin silicon layer is grown epitaxially using a low temperature $\left(<450{ }^{\circ} \mathrm{C}\right)$ molecular beam epitaxy process. This silicon epi grown layer is delta-doped during the growth process by incorporating a third of a monolayer of boron atoms, effectively as a single atomically thin sheet that does not interrupt the overall silicon epitaxy, 
but dramatically affects the electronic band structure of the silicon in the near surface region. This sheet becomes negatively charged and counteracts the positive potential well that typically forms at the surface due to the presence of silicon's native oxide. This allows the efficient collection of UV produced photoelectrons. This delta-layer enables the CCD to operate at $100 \%$ internal QE, limited only by reflection of light from the silicon surface.

AR coatings were tested to improve the imaging performance of these silicon CCDs. The index of refraction of the silicon changes significantly in the ultraviolet region of the spectrum, especially from 100 to $300 \mathrm{~nm}$. Therefore, to obtain optimum quantum efficiency, it was necessary to test different thin film materials and coating methods. Contact shadow masks, such as the one shown in Fig. 3, were positioned to ensure that all AR coated CCDs had an internal, uncoated standard for comparison to validate subsequent measurements of QE. The following thin film coating techniques were evaluated in this study: physical sputtering, electron beam evaporation, thermal evaporation, and atomic layer deposition.

Once the AR coatings were applied to the CCDs, they were packaged and tested in a custom built UV-visible CCD characterization system described elsewhere. ${ }^{18}$ Briefly, imaging performance of the CCD was characterized from 121.6 to $650 \mathrm{~nm}$ starting from the longer wavelength end of the spectrum by taking flat field images over controlled exposure times. Two separate light sources were used for illumination (deuterium lamp for UV and tungsten-halogen for UV-visible). Absolute QE measurement was made by comparison to a NIST calibrated photodiode that could be posi-

Thick silicon frame (protected during $\mathrm{KOH}$ and HNA etches by nitride hard mask)

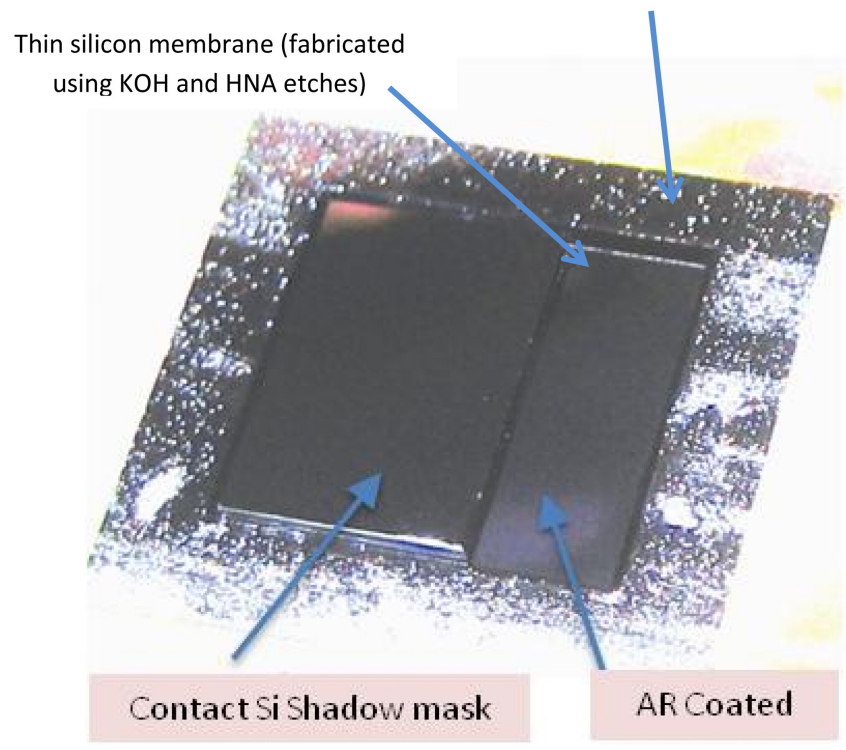

FIG. 3. (Color online) Demonstration of shadow masking approached used in AR coating experiments. A cleaved silicon wafer was placed in direct contact with the backside of a thin imaging membrane to block the deposition of the AR coating from a portion of the device. This shadow masking is used to ensure that coated and uncoated references were present within the same device. tioned in the same location as the CCD being characterized. Some of the CCD characterization data presented here (as noted on the figures in the text) was obtained in a different testing system of similar design.

\section{RESULTS AND DISCUSSION}

Thinned, delta-doped Cassini (1 megapixel, 12 micron pixels, manufactured by Loral Farchild) and EG\&G Reticon CCDs ( $1 \mathrm{kx} 1 \mathrm{k}, 7.5$ micron pixels) were utilized to evaluate thin film AR coating techniques and materials for their ability to improve absolute detector quantum efficiency. Magnesium oxide $(\mathrm{MgO})$ and hafnium oxide $\left(\mathrm{HfO}_{2}\right)$ were deposited by physical sputtering. $\mathrm{HfO}_{2} \mathrm{AR}$ coatings were also deposited by electron beam evaporation and thermal evaporation. Lastly, $\mathrm{HfO}_{2}$ and aluminum oxide $\left(\mathrm{Al}_{2} \mathrm{O}_{3}\right)$ were deposited by atomic layer deposition. This combination of materials and deposition methods was chosen to elucidate the efficacy of each deposition technique. Examples of flat field images obtained from some of the partially AR coated CCDs produced in this investigation are shown in Fig. 4. Figure 4 shows flat field images taken by two partially AR coated Cassini CCD under $300 \mathrm{~nm}$ UV illumination. The upper regions were sputter coated with $\mathrm{MgO}$ and $\mathrm{HfO}_{2}$, respectively, $\mathrm{A}$ and $\mathrm{B}$, while the lower regions were protected by the shadow mask. The relative performance of the coated and uncoated regions was counter to expectations as the brightness of these flat field images is directly related to the absolute quantum efficiency of the detector. As mentioned earlier, the short wavelength flat field brightness is enhanced because the quantum yield is greater than one, but in all cases, a brighter image indicates greater sensitivity. Figure 5 shows the quantum efficiency of these two CCDs over a range of wavelengths. The performance of the $\mathrm{MgO}$ coating was poor over the entire range of wavelengths. The $\mathrm{HfO}_{2}$ coating was roughly identical to the uncoated side in the range of visible wavelengths, but substantially dropped off in the ultraviolet. Figure 6 shows the $270 \mathrm{~nm}$ flat field image of an EG\&G Reticon CCD AR coated with various thicknesses of $\mathrm{HfO}_{2}$ in four different regions. The two circular regions of the device were AR coated using e-beam evaporation and the two square regions are AR coated using thermal evaporation. The circular AR coated regions of the device are believed to have failed due to the exposure of the $\mathrm{CCD}$ to $\mathrm{x}$-rays during the electron beam evaporation process. The brighter regions, coated by thermal evaporation of $\mathrm{HfO}_{2}$, demonstrate the successful application of a functioning AR coating. These results clearly indicate that, while delta-doped CCDs can be AR coated to improve their performance, the method of depositing the coating is critical.

Figure 7 shows the $183.2 \mathrm{~nm}$ flat field of a Cassini CCD AR coated with $\mathrm{Al}_{2} \mathrm{O}_{3}$ by plasma enhanced atomic layer deposition (PEALD). The demarcation between the brighter, AR coated region at the top, and the uncoated region at the bottom is blurred compared to the flat field images obtained for AR coatings applied using sputtering, e-beam evaporation, and thermal evaporation techniques. This is due to the highly conformal nature of the ALD process. The contact 
(a)

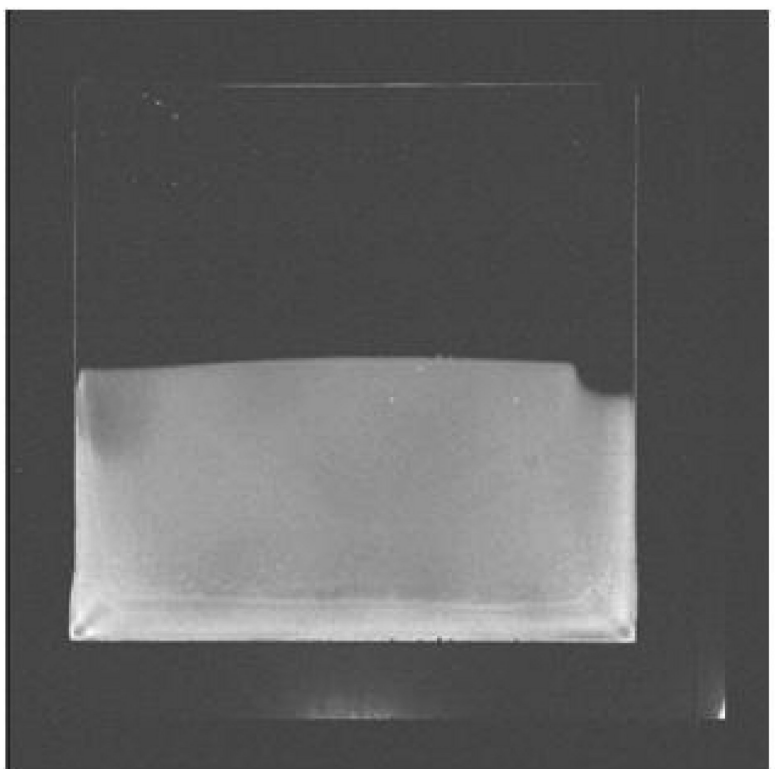

(b)

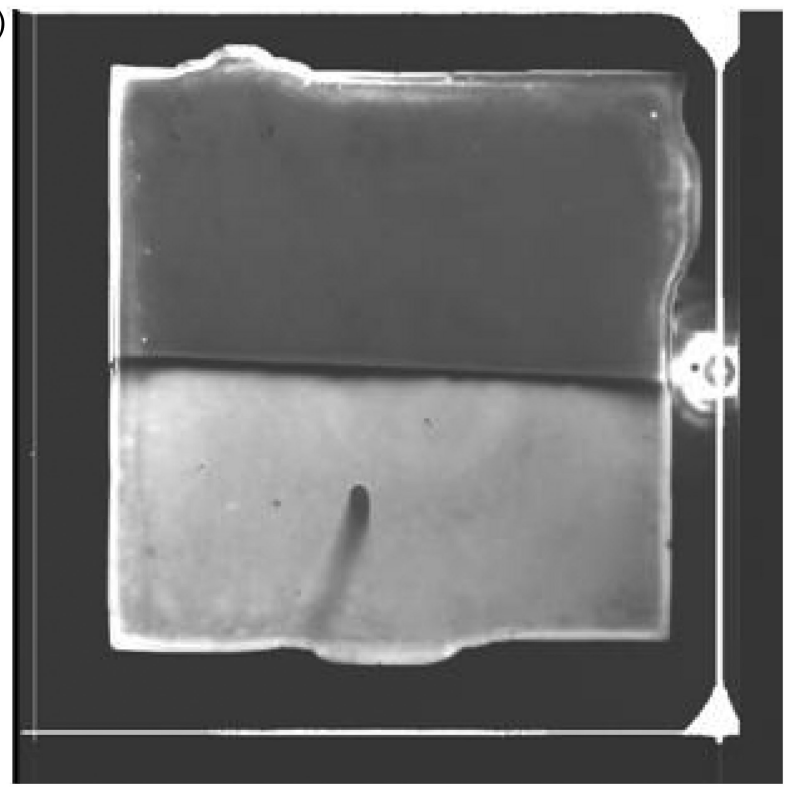

FIG. 4. Flat field images produced by delta-doped back-illuminated silicon CCDs when illuminated with $300 \mathrm{~nm}$ light. The top of each image comes from the half of the CCD that was coated with a sputtered film. (a) CCD half coated with sputtered $\mathrm{MgO}$ film. (b) CCD half coated with sputtered $\mathrm{HfO}_{2}$ film. The brightness of the image is proportional to the QE. Note that in each case, the side of the CCD that is sputter coated demonstrates lower quantum efficiency than the uncoated side.

shadow masking technique (shown in Fig. 3) has topographic limitations due to the compressive stresses present in the thin silicon membrane. The raised clover-leaf pattern in the membrane (shown in Fig. 2) causes the shadow mask to be elevated above a certain portion of the imaging surface. That allows the ALD process to partially coat the shadowed regions because the ALD precursors can penetrate under the mask. However, this is not a concern for the real world applicability of these techniques. It is noted here again that the purpose of the shadow mask is only to provide an internal calibration standard for an uncoated delta-doped CCD.

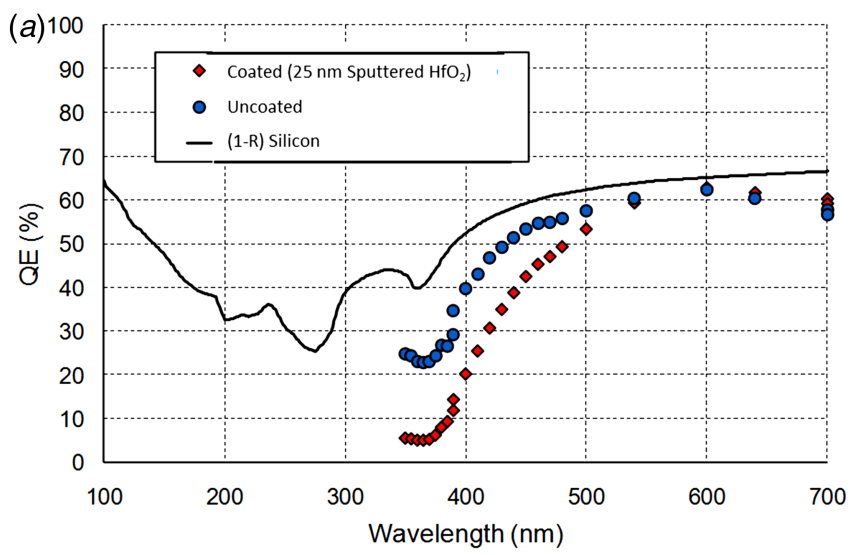

(b)

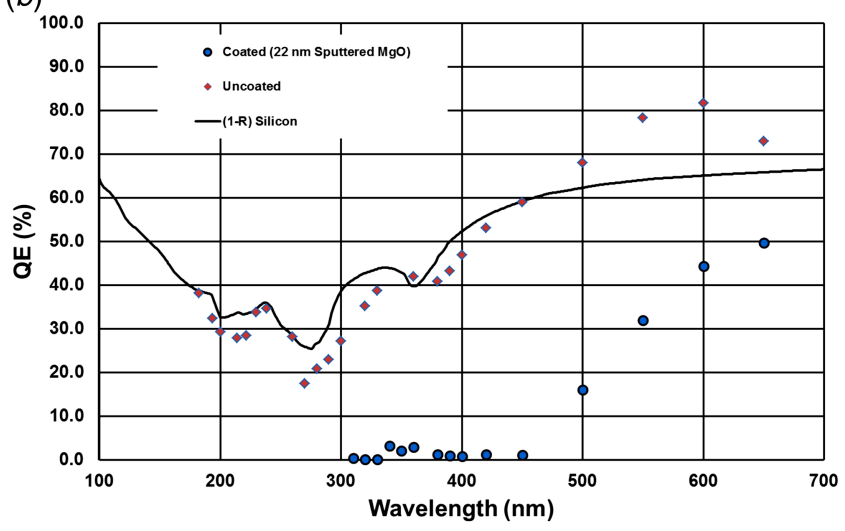

FIG. 5. (Color online) Quantum efficiency for the AR coated and uncoated sides of delta-doped CCDs. The AR coatings were applied by sputter deposition [(a): $\left.\mathrm{HfO}_{2},(\mathrm{~b}): \mathrm{MgO}\right]$. Note that the quantum efficiency of the coated side of the CCD has worse performance, contrary to the calculations made using TF-calc ${ }^{\mathrm{TM}}$ that had indicated the utility of $\mathrm{HfO}_{2}$ and $\mathrm{MgO}$ at those thicknesses.

The ultimate applications for these UV AR Coatings will most likely require uniform blanket coatings over an entire $\mathrm{CCD}$, eliminating the shadow masking artifacts.

The design and accurate prediction of the performance of ALD AR coatings was straightforward due to the inherent reproducibility and control of the ALD technique. Figure 8(a) shows the performance of a conventional and EM-CCD coated with a $16.5 \mathrm{~nm}$ AR coating of aluminum oxide. Their measured quantum efficiency is the same both qualitatively and quantitatively over the entire wavelength range tested despite there being a month between deposition runs. The modeled performance of the coatings also matches the measured data extremely well. Figure 8(b) shows that a $23 \mathrm{~nm}$ aluminum oxide, deposited by scaling to the appropriate number of ALD cycles based on the $16.5 \mathrm{~nm}$ result, displays quantum efficiency that qualitatively matches that of the predicted values. The absolute $\mathrm{QE}$ is a bit lower than the modeled value, as the absorption parameters used in the $\mathrm{TF}-\mathrm{calc}^{\mathrm{TM}}$ model are taken from the literature for bulk materials rather than the films in this study.

While $\mathrm{HfO}_{2}$ AR coatings deposited by thermal evaporation do perform well, the ALD technique has inherent advantages in uniformity and reproducibility, which are of great importance in the production of high quality AR coatings. These advantages make it generally preferable to use to 


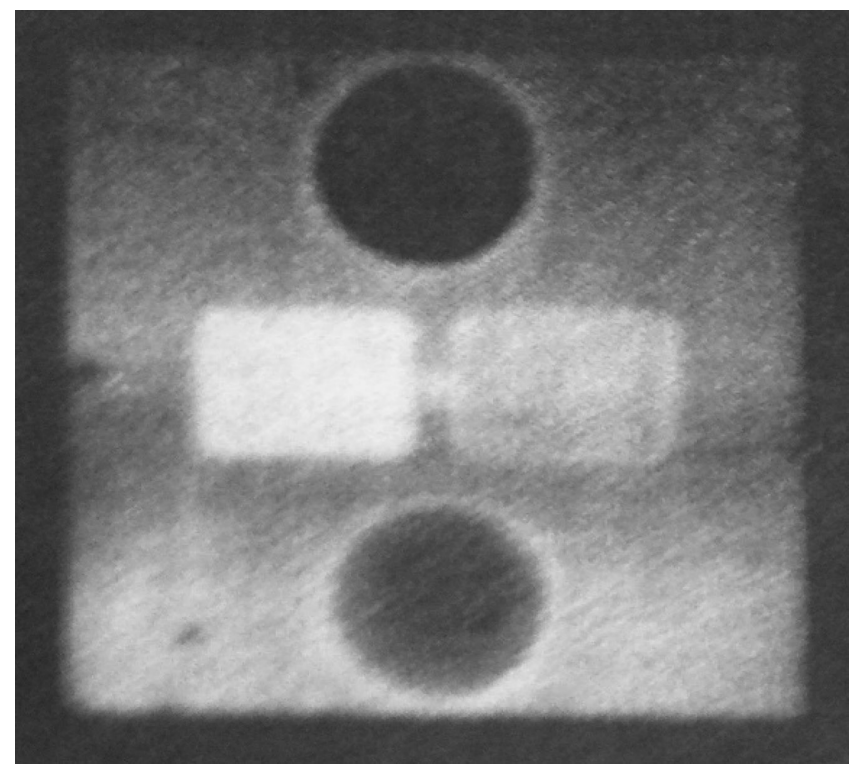

FIG. 6. Flat field image taken under $270 \mathrm{~nm}$ illumination. The dark circles at the top and bottom of the image are regions that were coated with $\mathrm{HfO}_{2}$ using electron beam evaporation. It is believed that $\mathrm{x}$-ray damage during the evaporation degraded the CCDs imaging capability in these regions. The bright rectangular regions are coated with $\mathrm{HfO}_{2}$ using thermal evaporation. The differences in the brightness of the two regions are due to different thicknesses of AR coating applied to each ( 25 and $40 \mathrm{~nm}$, respectively). Changing AR coating thickness enables one to maximize performance at different target wavelength ranges (see Fig. 14).

ALD instead of thermal evaporation for these ultrathin UV-AR coatings. However, since remote plasma ALD of hafnium oxide on hydrogen terminated silicon is known to lead to the formation of a hafnium silicate interfacial layer, transmission electron microscopy was utilized to determine whether a similar silicate would form during $\mathrm{HfO}_{2}$ deposition on a delta doped silicon wafer. ${ }^{23}$ Note that there is an air exposure between the delta-doping process and the deposi-

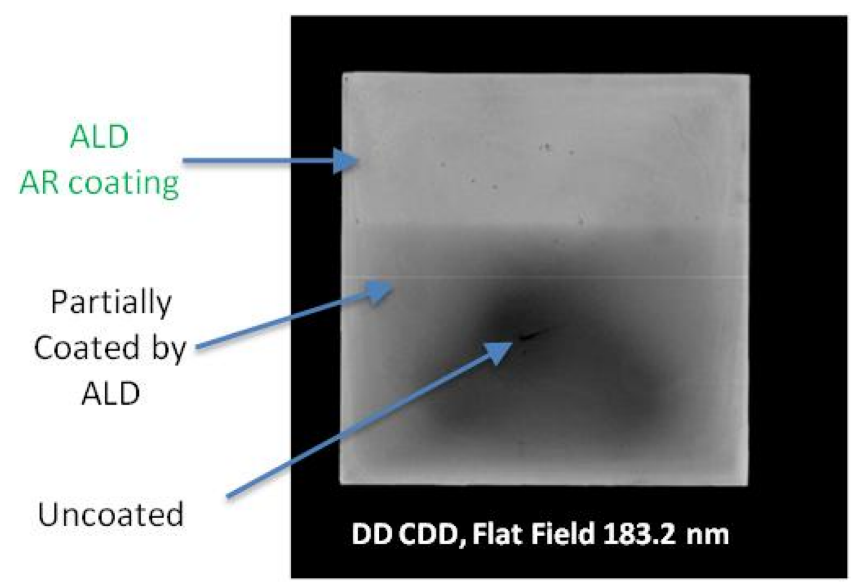

FIG. 7. (Color online) Flat field image produced by delta-doped AR coated back-illuminated silicon CCD when illuminated with $183.2 \mathrm{~nm}$ light. The top of this image comes from the portion of the CCD that is coated with a PE-ALD $\mathrm{Al}_{2} \mathrm{O}_{3}$ film. The brightness of the image is proportional to the QE. Note that the side of the CCD that is AR coated has higher quantum efficiency than the uncoated side. The shadow masking used is imperfect for this device due to the highly conformal nature of ALD deposition processes, which allows the coating to sneak under the shadow mask (see Figs. 2 and 3).

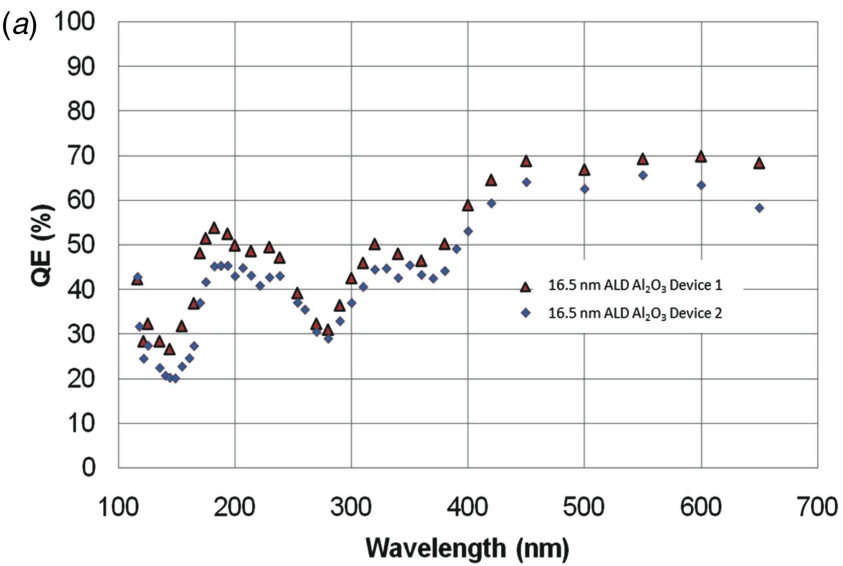

(b)

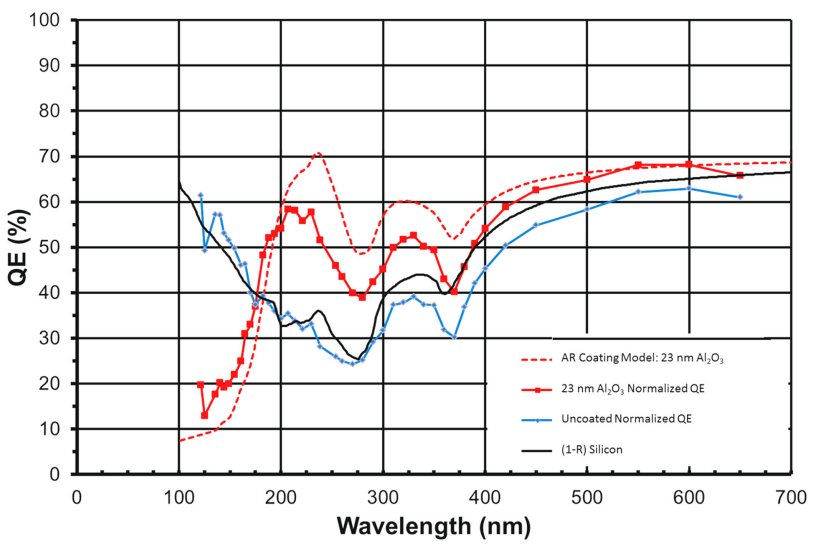

FIG. 8. (Color online) Demonstration of the repeatability and control of the ALD technique. (a) Quantum efficiency for two individual delta-doped$\mathrm{CCD}$ soated on one half by $16.5 \mathrm{~nm}$ thick ALD $\mathrm{Al}_{2} \mathrm{O}_{3}$ AR coating. Device \#1 was a conventional CCD, while device \#2 is an electron multiplied CCD with gain. Note that there is no measurable quantitative or qualitative difference between the two devices when the measurement error $(+/-5 \%)$ is taken into account. The depositions were carried out using the same deposition recipe but were separated by a month between coatings. (b) Comparison of modeled and measured performance of a $23 \mathrm{~nm}$ thick ALD $\mathrm{Al}_{2} \mathrm{O}_{3}$ AR coated CCD. Note that the qualitative $\mathrm{QE}$ behavior predicted by our model matches the measured performance.

tion of the ALD AR coating, so a native oxide silicon oxide is present prior to the ALD process. The TEM image in Fig. 9 shows that a large interfacial layer has formed between the ALD $\mathrm{HfO}_{2}$ (the dark layer at the top of the image) and the crystalline silicon substrate. Because this undesirable interfacial layer was observed, a $2 \mathrm{~nm}$ ALD aluminum oxide film was deposited in situ as a barrier, immediately prior to the ALD $\mathrm{HfO}_{2}$ deposition during all further ALD $\mathrm{HfO}_{2}$ AR coating experiments. A high resolution TEM image of this ALD bilayer is shown at left in Fig. 10. This image confirms that a high density, nanocrystalline $\mathrm{HfO}_{2}$ film is produced and chemical interactions between the $\mathrm{HfO}_{2}$ and the silicon are blocked.

X-ray photoelectron spectroscopy (XPS) was employed to characterize the nature of the silicon/ALD interface whether the $2 \mathrm{~nm}$ aluminum oxide layer was sufficient to prevent the formation an interfacial hafnium silicate layer. Samples were prepared where 25 ALD cycles $(\sim 1.2 \mathrm{~nm})$ of $\mathrm{HfO}_{2}$ were deposited on $2 \mathrm{~nm}$ of $\mathrm{Al}_{2} \mathrm{O}_{3}$ on silicon. Figure 11 shows the high resolution XPS scans for hafnium $4 \mathrm{f}$ peaks for these two 


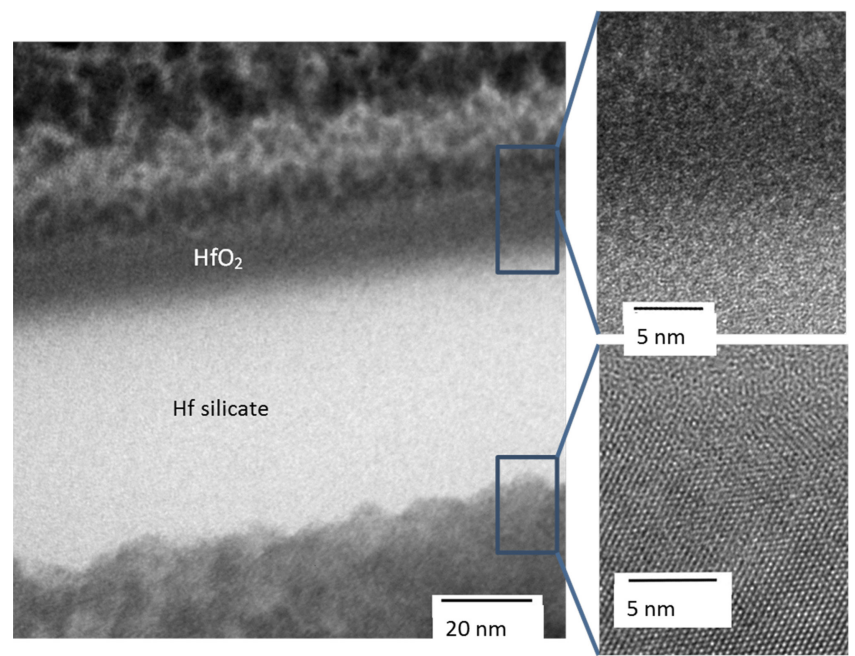

FIG. 9. (Color online) TEM image of PEALD $\mathrm{HfO}_{2}$ grown on a blank deltadoped silicon wafer. Note that the PEALD process forms an interfacial layer despite the presence of the native oxide that forms after the delta-doping process. The prevention of the formation of this silicate layer provides the motivation for the ultrathin $(2 \mathrm{~nm})$ aluminum oxide barrier layer grown for the $\mathrm{HfO}_{2}$ AR coatings in this study.

samples. The absence of the low binding energy tail for the $\mathrm{HfO}_{2}$ grown on the $2 \mathrm{~nm} \mathrm{Al}_{2} \mathrm{O}_{3}$ barrier layer on silicon confirms the TEM result that $2 \mathrm{~nm}$ is sufficient to prevent the formation of interfacial hafnium silicates. Figure 11 also shows data for the sputtered hafnium oxide film. The sputtered hafnium $4 \mathrm{f}$ results appear to be comparable to the ALD bilayer, which suggests that the poor performance of the sputtered hafnium oxide [shown in Fig. 5(a)] is not related to a chemical interaction between the coating and the silicon surface of the CCD. This conclusion is supported by high resolution TEM analysis of a sputtered $\mathrm{HfO}_{2}$ film on delta-doped silicon (Fig. 12). Unlike the case of PEALD $\mathrm{HfO}_{2}$ deposited directly

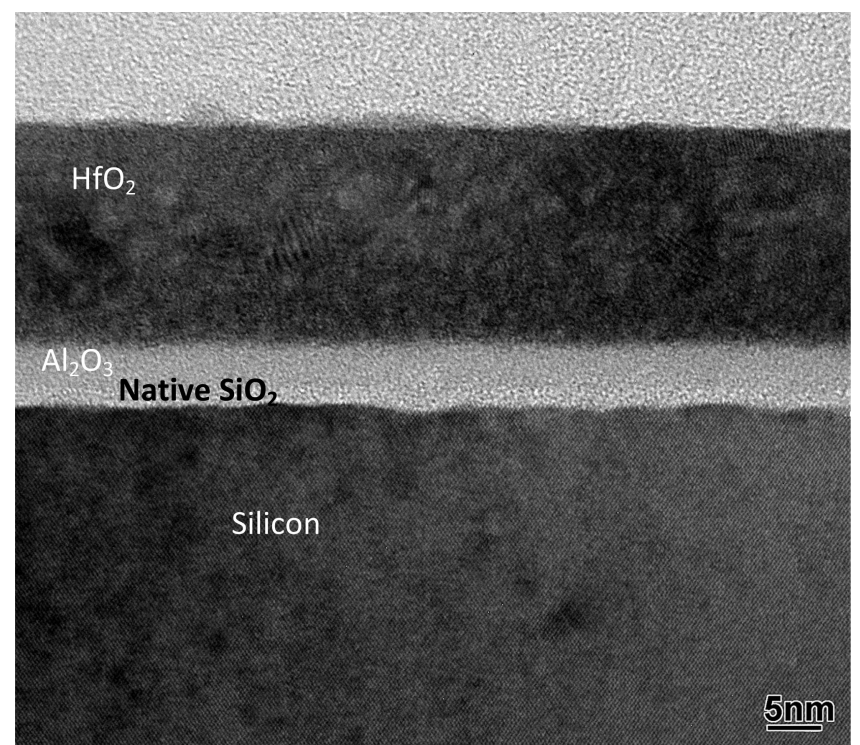

FIG. 10. TEM images of a PEALD $\mathrm{HfO}_{2} / \mathrm{Al}_{2} \mathrm{O}_{3}$ bilayer grown on a blank delta-doped silicon wafer. Note that the presence of the thin $(2 \mathrm{~nm})$ aluminum oxide barrier layer prevents the formation of the interfacial layer observed Fig. 9.

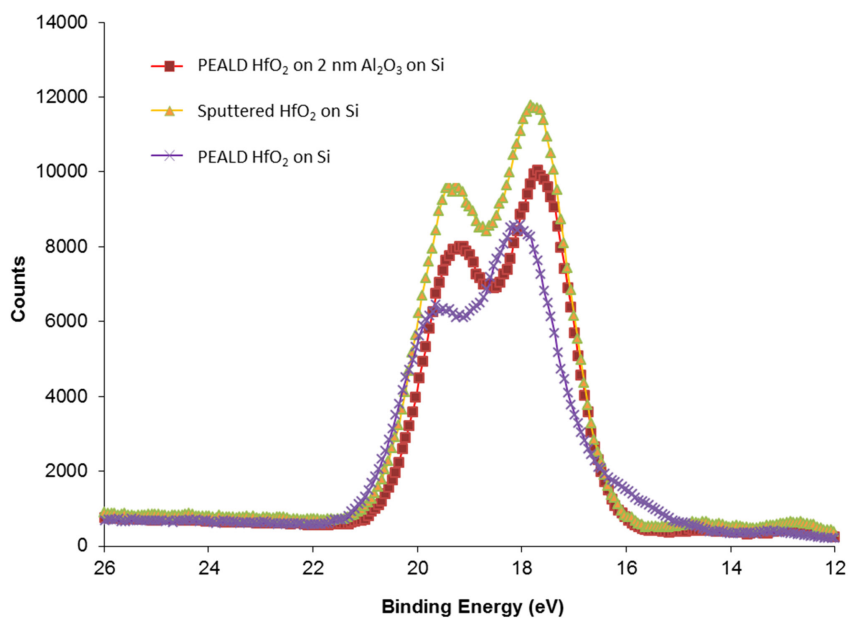

FIG. 11. (Color online) High resolution hafnium 4f XPS scans for three hafnium oxide AR coatings. The samples were prepared to target $\mathrm{HfO}_{2}$ film thicknesses of $\sim 1.2 \mathrm{~nm}$ in all cases to enable the XPS to characterize the interface properties. The sputtered $\mathrm{HfO}_{2}$ deposited on silicon and the PEALD $\mathrm{HfO}_{2}$ grown on the $2 \mathrm{~nm}$ barrier layer of $\mathrm{Al}_{2} \mathrm{O}_{3}$ show $\mathrm{Hf}$ peaks consistent with the data observed for much thicker films. However, the presence of a low binding energy tail for the PEALD $\mathrm{HfO}_{2}$ grown directly on silicon suggests that a chemical interaction has occurred between of the $\mathrm{HfO}_{2}$ and the underlying silicon (see TEM in Fig. 9).

on silicon, there is no evidence of a large silicate interfacial layer. The lighter layer at the boundary is likely the native oxide that forms after the delta-doping process prior to the AR coating deposition. However, the sputtered $\mathrm{HfO}_{2}$ film appears noticeably less dense, amorphous, and rough as compared to the corresponding PEALD $\mathrm{HfO}_{2}$ bilayer (see Fig. 11). This may account for the superior performance of the PEALD bilayer as an AR coating.

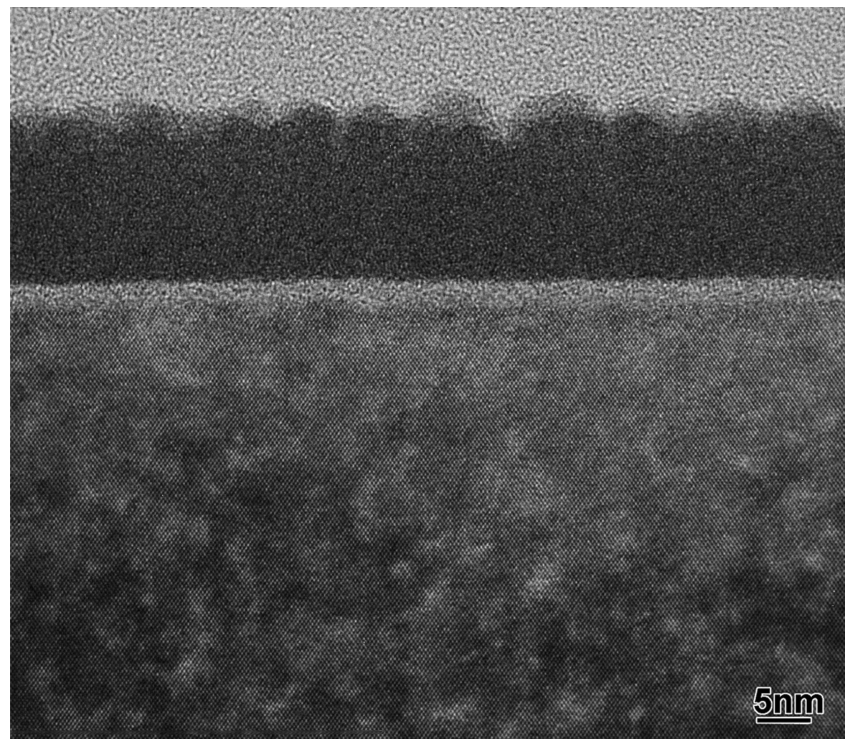

FIG. 12. TEM images of a sputtered $\mathrm{HfO}_{2}$ thin film on a blank delta-doped silicon wafer. Unlike the case of PEALD $\mathrm{HfO}_{2}$ deposited directly on silicon, there is no evidence of a large silicate interfacial layer. The lighter layer at the boundary is likely the native oxide that forms after the delta-doping process. The sputtered $\mathrm{HfO}_{2}$ film appears less dense, amorphous, and rough as compared to the corresponding PEALD $\mathrm{HfO}_{2}$ bilayer (see Fig. 10). 
Figure 13 shows the optical properties of the $\mathrm{HfO}_{2}$ ALD AR single and bilayer coatings as compared to the sputtered $\mathrm{HfO}_{2}$ thin films. These three coatings were deposited on 1 in. diameter silicon substrates, and the optical properties were calculated by J.A. Woollam Inc. using ultraviolet spectroscopic ellipsometry. The roughness values, as estimated by the model fits, for the two ALD samples were substantially lower than the sputtered $\mathrm{HfO}_{2}$ sample $(1.3 \mathrm{~nm}$ vs $3.5 \mathrm{~nm}$ ). The superiority of the aluminum oxide/hafnium oxide ALD bilayer as compared $\mathrm{HfO}_{2}$ alone is clear both from the effective index of refraction of the deposited films and the lower absorption coefficient. This supports the conclusion that the hafnium component of the PEALD bilayer stack has a higher density than the sputtered film. It also is

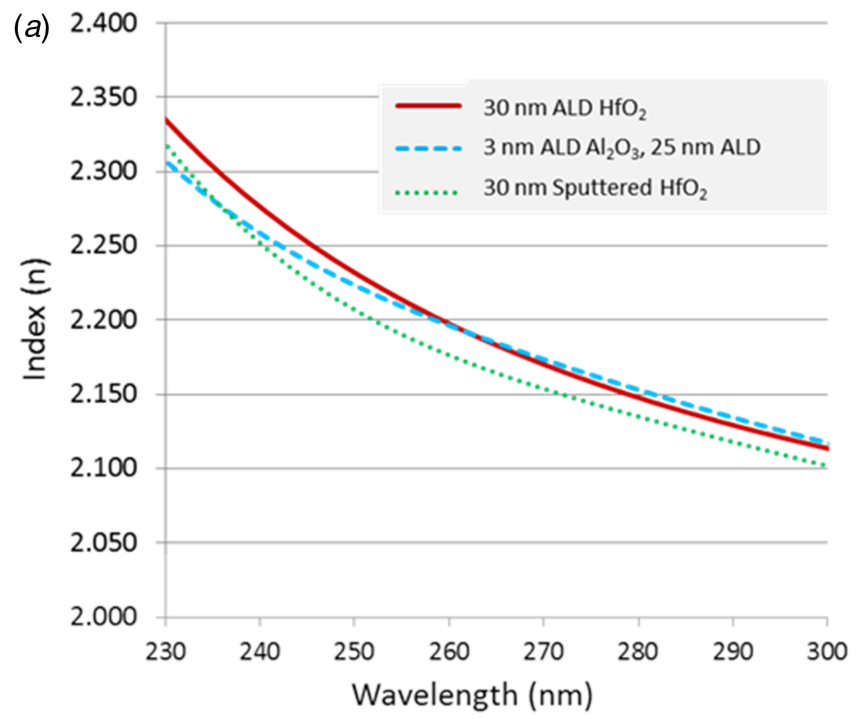

(b)

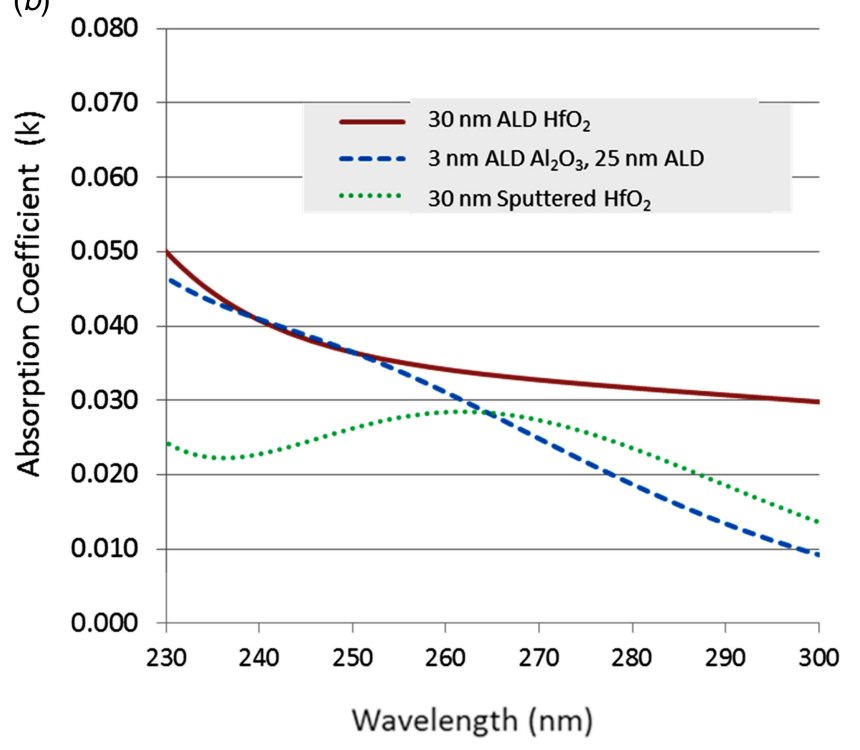

FIG. 13. (Color online) Optical properties of ALD and sputter deposited AR coatings. (a) Index of refraction of $\mathrm{HfO}_{2}$ and $\mathrm{HfO}_{2} / \mathrm{Al}_{2} \mathrm{O}_{3}$ bilayer coatings. Note that the ALD coatings have higher index of refraction over the NUV band (above $235 \mathrm{~nm}$ ), which is indicative of a higher quality thin film as it is closer to the value for bulk $\mathrm{HfO}_{2}$. (b) Absorption coefficient, as modeled by UV spectroscopic ellipsometry for $\mathrm{HfO}_{2}$ and $\mathrm{HfO}_{2} / \mathrm{Al}_{2} \mathrm{O}_{3}$ bilayer AR coatings. Note that the ALD bilayer shows the lowest absorption over the wavelength range of $265-300 \mathrm{~nm}$.

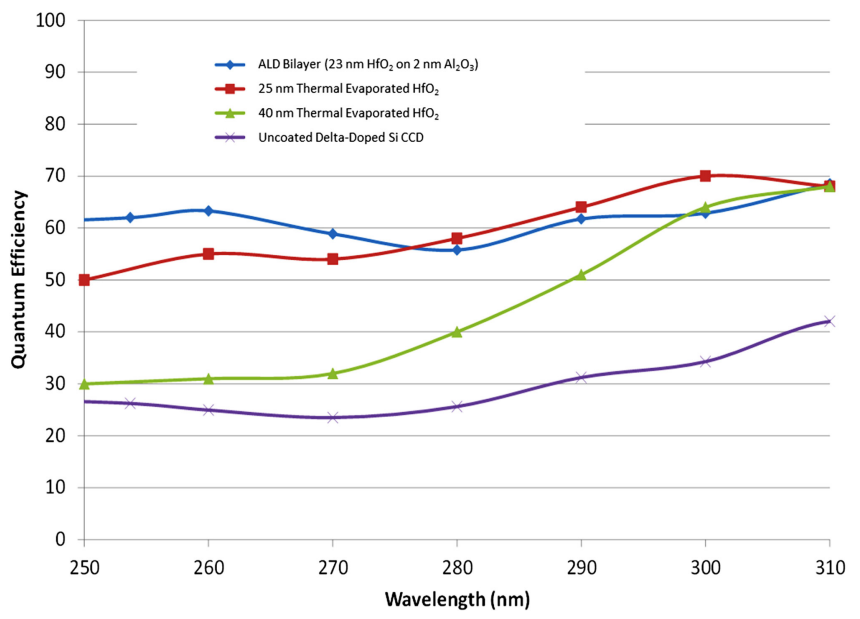

FIg. 14. (Color online) Comparison of $\mathrm{HfO}_{2} \mathrm{AR}$ coated delta-doped CCDs. The ALD bilayer outperforms the thermally evaporated $\mathrm{HfO}_{2}$ coatings at shorter wavelengths. This may be due to superior film quality, or the slight difference in thickness between the $23 \mathrm{~nm}$ ALD and $25 \mathrm{~nm}$ evaporated films. The sputter deposited $\mathrm{HfO}_{2}$ data are not shown here, due to its negligible UV response.

consistent with the XPS observation that the $\mathrm{HfO}_{2}$ in the PEALD bilayer has not reacted with the silicon substrate.

Figure 14 compares the quantum efficiency of $\mathrm{HfO}_{2} \mathrm{AR}$ coatings produced by thermal evaporation and PEALD. The QE of the ALD bilayer is somewhat higher than the thermally evaporated $\mathrm{HfO}_{2}$ coatings at shorter wavelengths. This may be due to differences in the film properties, or the slight difference in thickness between the $23 \mathrm{~nm}$ ALD and $25 \mathrm{~nm}$ evaporated films. The sputter deposited $\mathrm{HfO}_{2}$ data are not shown here, due to its negligible UV response. The TEM, XPS, and ellipsometry observations discussed above provide insight into the mechanisms for why the quantum efficiency of the sputtered AR coatings is degraded in comparison to the other techniques. Although the differences in optical constants appear to be relatively minor between these different techniques, it appears that the roughness and lower density of the sputtered $\mathrm{HfO}_{2}$ is sufficient to dramatically impact the AR coating performance, perhaps due to scattering of the incident UV light. It is possible that by employing a small amount of bias to the substrate during to smooth and density the film would help improve the quality of the sputtered $\mathrm{HfO}_{2}$ AR coating.

Figure 15 shows the quantum efficiency measured for the three different ALD AR coatings characterized in this study. For clarity, the performance of only one of the uncoated sides of these is presented as a reference for all three CCDs. The thicknesses of these ALD coatings were selected to maximize quantum efficiency over this range, dividing the spectra into three different near ultraviolet (NUV) and FUV bands. The bands selected were as follows: $170-200 \mathrm{~nm}$, 190-240 nm, and 230-300 nm. Through judicious choices of these materials and their thicknesses, the absolute QE can be maintained above $50 \%$ for the entire range from 170 to $300 \mathrm{~nm}$. With more complex coatings (multilayers or graded materials), it should be possible to obtain QE's $\sim 90 \%$ or greater in certain regions of the spectrum. Therefore, there is a great deal of promise in this AR coating technique. 


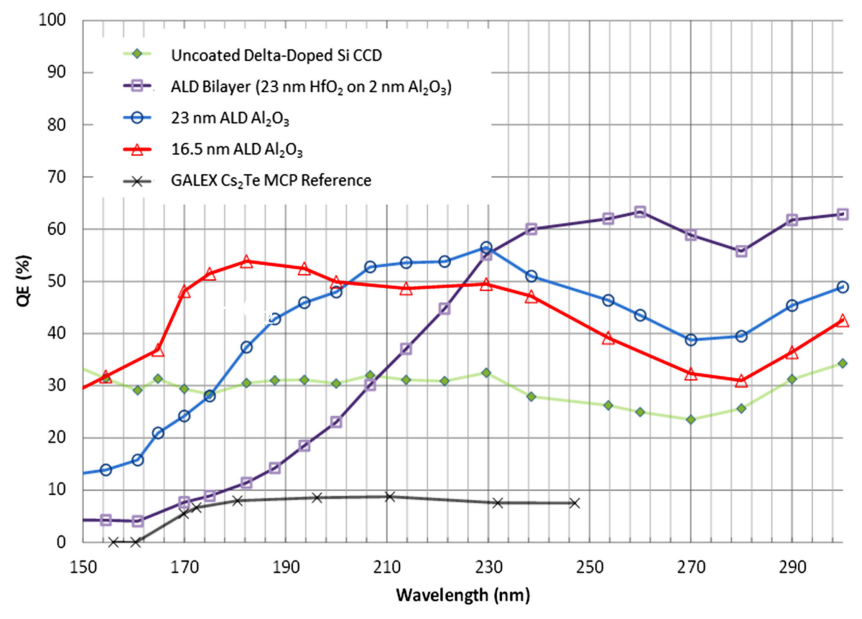

FIG. 15. (Color online) Quantum efficiency for AR coated delta dopedCCDs compared against that of the uncoated portion of a delta doped-CCD and the multichannel plate onboard GALEX. Film type and thicknesses have been selected to optimize $\mathrm{QE}$ in defined wavelength regions. These wavelength regions are $170-200 \mathrm{~nm}\left(16.5 \mathrm{~nm} \mathrm{Al}_{2} \mathrm{O}_{3}\right), 190-240 \mathrm{~nm}(23 \mathrm{~nm}$ $\left.\mathrm{Al}_{2} \mathrm{O}_{3}\right)$, and $230-300 \mathrm{~nm}\left(23 \mathrm{~nm} \mathrm{HfO} \mathrm{H}_{2} / 2 \mathrm{~nm} \mathrm{Al}_{2} \mathrm{O}_{3}\right)$ and were chosen based on the index of available UV transparent materials.

\section{SUMMARY AND CONCLUSIONS}

The unique challenges presented by imaging in the ultraviolet require surface engineering at the nanoscale to control both the electronic band structure and optical properties of the silicon-based UV detector surface. In this paper, several different AR coatings and materials deposition methodologies were evaluated experimentally for their ability to optimize detector sensitivity. By combining the atomic precision of molecular beam epitaxy and atomic layer deposition, a robust and reproducible technique was developed to fabricate a new class of delta-doped silicon CCD ultraviolet imaging detectors demonstrating world record quantum efficiency in the near and far UV (155-300 nm). This new demonstration of the integration of ALD coatings onto live silicon detectors opens tremendous possibilities in tailoring the response of detectors. Given the variety of materials available to be deposited by ALD, this technique offers great flexibility in tailoring the exact sensitivity and responsiveness of a detector to any given application. As ultraviolet imaging systems have the potential to detect life on other planets, explain the origins of the universe, identify defects in the latest generation of semiconductor devices, perform medical imaging and brain mapping, improve missile defense, and even solve crimes, this new approach to UV detector fabrication may have an extremely significant impact on a wide range of fields.

\section{ACKNOWLEDGMENTS}

The research described in this paper was carried out at the Jet Propulsion Laboratory, California Institute of Technology, under a contract with the National Aeronautics and Space Administration. The authors gratefully acknowledge the work of the J.A. Woollam company to provide UV ellipsometry data for the films described here. The authors also gratefully acknowledge the support of the Keck Institute for Space Studies for facilitating helpful discussions between the authors and other attendees of the recent workshop entitled: Next Generation UV Instrument Technologies.

${ }^{1}$ C. A. Barth, Appl. Optics 8, 1295 (1969).

${ }^{2}$ A. R. Hendrix, C. J. Hansen, and G. M. Holsclaw, Icarus 206, 608 (2010).

${ }^{3}$ F. Nicastro, S. Mathur, and M. Elvis, Science 319, 55 (2008).

${ }^{4}$ D. C. Martin et al., Nature 448, 780 (2007).

${ }^{5}$ Y. D. Chan, A. Rastegar, H. Yun, E. S. Putna, and S. Wurm, Proc. SPIE 76361 (2010).

${ }^{6}$ T. D. Tsai and M. E. Barish, J. Neurobiol. 27, 252 (1995).

${ }^{7}$ D. A. Levin, Proc. SPIE 1764, 388 (1993).

${ }^{8}$ M. H. West, J. D. Billings, and J. Fair, J. Forensic Sci. 32, 1204 (1987).

${ }^{9}$ C. L. Joseph, Proc. SPIE 3764, 246 (1999).

${ }^{10}$ C. C. Porco et al., Space Sci. Rev. 115, 363 (2004).

${ }^{11}$ M. E. Hoenk, P. J. Grunthaner, F. J. Grunthaner, R. W. Terhune, M. Fattahi, and H.-F. Tseng, Appl. Phys. Lett. 61, 1084 (1992).

${ }^{12}$ M. P. Lesser, Proc. SPIE 4139, 8 (2000).

${ }^{13}$ M. C. Peckerar, J. T. Bosiers, D. McCarthy, N. S. Saks, and D. J. Michels, Appl. Phys. Lett. 50, 1275 (1987).

${ }^{14}$ M. P. Lesser, Proc. SPIE 2198, 782 (1994).

${ }^{15}$ B. E. Burke, J. A. Gregory, A. H. Loomis, S. D. Calawa, P. M. Nitishin, T. A. Lind, M. J. Cooper, D. J. Young, P. W. O'Brien, B. B. Kosicki, G. A. Luppino and J. L. Tonry, Scientific Detectors for Astronomy, The Beginning of a New Era, edited by P. Amico, J. W. Beletic, and J. E. Beletic (2004), Vol. 300, p. 41-49.

${ }^{16}$ S. Nikzad, M. E. Hoenk, P. J. Grunthaner, R. W. Terhune, F. J. Grunthaner, R. Winzenread, M. M. Fattahi, and H.-F. Tseng, Proc. SPIE 2278, 138 (1994).

${ }^{17}$ E. T. Hamden, F. Greer, M. E. Hoenk, J. Blacksberg, M. R. Dickie, S. Nikzad, D. C. Martin, and D. Schiminovich., Applied Optics 50, 4180 (2011).

${ }^{18}$ B. C. Jacquot, S. P. Monacos, M. E. Hoenk, F. Greer, T. J. Jones, and S. Nikzad, Rev Sci Instrum 82, 043102 (2011).

${ }^{19}$ C. C. Porco, Science 299, 1541 (2003).

${ }^{20}$ H. Robbins and B. Schwartz, J. Electrochem. Soc. 106, 505 (1959).

${ }^{21}$ S. Nikzad, T. J. Jones, S. T. Elliott, T. J. Cunningham, P. W. Deelman, A. B. C. Walker II, and H. M. Oluseyi, Proc. SPIE 4139, 250 (2000).

${ }^{22}$ S. Nikzad, M. E. Hoenk, P. J. Grunthaner, R. W. Terhune, F. J. Grunthaner, R. Winzenread, M. M. Fattahi, H.-F. Tseng, and M. P. Lesser, Proc. SPIE 2198, 907 (1994).

${ }^{23}$ Y. Won, S. Park, J. Koo, S. Kim, J. Kim, and H. Jeon, Appl. Phys. Lett. 87, 262901 (2005). 